\title{
A SURVEY OF SOME RECENT DEVELOPMENTS IN DIFFERENTIAL TOPOLOGY
}

\author{
S. SMALE
}

1. We consider differential topology to be the study of differentiable manifolds and differentiable maps. Then, naturally, manifolds are considered equivalent if they are diffeomorphic, i.e., there exists a differentiable map from one to the other with a differentiable inverse. For convenience, differentiable means $C^{\infty}$; in the problems we consider, $C^{\prime}$ would serve as well.

The notions of differentiable manifold and diffeomorphism go back to Poincaré at least. In his well-known paper, Analysis situs [27] (see pp. 196-198), topology or analysis situs for Poincaré was the study of differentiable manifolds under the equivalence relation of diffeomorphism. Poincaré used the word homeomorphism to mean what is called today a diffeomorphism (of class $C^{\prime}$ ). Thus differential topology is just topology as Poincaré originally understood it.

Of course, the subject has developed considerably since Poincaré; Whitney and Pontrjagin making some of the major contributions prior to the last decade.

Slightly after Poincaré's definition of differentiable manifold, the study of manifolds from the combinatorial point of view was also initiated by Poincaré, and again this subject has been developing up to the present. Contributions here were made by Newman, Alexander, Lefschetz and J. H. C. Whitehead, among others.

What started these subjects? First, it is clear that differential geometry, analysis and physics prompted the early development of differential topology (it is this that explains our admitted bias toward differential topology, that it lies close to the main stream of mathematics). On the other hand, the combinatorial approach to manifolds was started because it was believed that these means would afford a useful attack on the differentiable case. For example, Lefschetz wrote $[13$, p. 361], that Poincaré tried to develop the subject on strictly "analytical" lines and after his Analysis situs, turned to combinatorial methods because this approach failed for example in his duality theorem.

Naturally enough, mathematicians have been trying to relate these

An address delivered before the Stillwater meeting of the Society on August 31, 1961, by invitation of the Committee to Select Hour Speakers for Western Sectional Meetings; received by the editors November 28, 1962. 
two viewpoints that have developed side by side. S. S. Cairns is an example of such.

In the last decade, the three domains, differential topology, combinatorial study of manifolds, and the relations between the two, have all advanced enormously. Of course, these developments are not isolated from each other. However, we would like to make the following important point.

It has turned out that the main theorems in differential topology did not depend on developments in combinatorial topology. In fact, the contrary is the case; the main theorems in differential topology inspired corresponding ones in combinatorial topology, or else have no combinatorial counterpart as yet (but there are also combinatorial theorems whose differentiable analogues are false).

Certainly, the problems of combinatorial manifolds and the relationships between combinatorial and differentiable manifolds are legitimate problems in their own right. An example is the question of existence and uniqueness of differentiable structures on a combinatorial manifold. However, we don't believe such problems are the goal of differential topology itself. This view seems justified by the fact that today one can substantially develop differential topology most simply without any reference to the combinatorial manifolds.

We have not mentioned the large branch of topology called homotopy theory until now. Homotopy theory originated as an attack on the homeomorphism or diffeomorphism problem, witness the "Poincare Conjecture" that the homotopy groups characterize the homeomorphism type of the 3-sphere, and the Hurewicz conjecture that the homeomorphism type of a closed manifold is determined by the homotopy type. One could attack the homotopy problem more easily than the homeomorphism one and, for many years, most of the progress in topology centered around the homotopy problem.

The Hurewicz conjecture turned out not to be true, but amazingly enough, as we shall see, the last few years have brought about a reduction of a large part of differential topology to homotopy theory. These problems do not belong so much to the realm of pure homotopy theory as to a special kind of homotopy theory connected with vector space bundles and the like, as exemplified by work around the Bott periodicity theorems.

Of course, there are a number of important problems left in differential topology that do not reduce in any sense to homotopy theory and topologists can never rest until these are settled. But, on the other hand, it seems that differential topology has reached such a satisfactory stage that, for it to continue its exciting pace, it must 
look toward the problems of analysis, the sources that led Poincare to its early development.

We here survey some developments of the last decade in differential topology itself. Certainly, we make no claims for completeness. A notable omission is the work of Thom, on cobordism, and the study of differentiable maps. The reader is referred to expositions of Milnor [21] and H. Levine [14] for accounts of part of this work.

2. We now discuss what must be considered a fundamental problem of differential topology, namely, the diffeomorphism classification of manifolds.

The classification of closed orientable 2-manifolds goes back to Riemann's time. The next progress on this problem was the development of numerical and algebraic invariants which were able to distinguish many nondiffeomorphic manifolds. These invariants include, among others, the dimension, betti numbers, homology and homotopy groups and characteristic classes.

For dimension greater than two, there was still (at the beginning of 1960) no known case where the existing numerical and algebraic invariants determined the diffeomorphism class of the manifold. The simplest case of this problem (or so it appeared) was that posed by Poincaré: Is a 3-manifold which is closed and simply connected, homeomorphic (equivalently diffeomorphic) to the 3-sphere? This has never been answered.

The surprising thing is, however, that without resolving this problem, the author showed that in many cases, the known numerical and algebraic invariants were sufficient to characterize the diffeomorphism class of a manifold. Generally speaking in fact, considerable information on the structure of manifolds was found. We will now give an account of this.

To see how manifolds can be constructed, one defines the notion of attaching a handle. Let $M^{n}$ be a compact manifold with boundary $\partial M$ (we remind the reader that everything is considered from the $C^{\infty}$ point of view, manifolds, imbeddings, etc.) and let $D^{s}$ be the $s$-disk (i.e., the unit disk of Euclidean $s$-space $\left.E^{s}\right)$. Suppose $f:\left(\partial D^{s}\right) \times D^{n-s}$ $\rightarrow M$ is an imbedding. Then $X(M ; f ; s)$, " $M$ with a handle attached by $f$," is defined by identifying points under $f$ and imposing a differentiable structure on $M U_{f} D^{s} \times D^{n-s}$ by a process called "straightening the angle." Similarly if $f_{i}:\left(\partial D_{i}^{s}\right) \times D_{i}^{n-s} \rightarrow M, i=1, \cdots, k$, are imbeddings with disjoint images one can define $X\left(M ; f_{1}, \cdots, f_{k} ; s\right)$. If $M$ itself is a disk, then $X\left(M ; f_{1}, \cdots, f_{k} ; s\right)$ is called a handlebody.

(2.1) TheOREM. Let $f$ be a $C^{\infty}$ function on a closed (i.e. compact with 
empty boundary) manifold with no critical points on $f^{-1}[-\epsilon, \epsilon]$ except $k$ nondegenerate ones on $f^{-1}(0)$, all of index s. Then $f^{-1}[-\infty, \epsilon]$ is diffeomorphic to $X\left(f^{-1}[-\infty,-\epsilon] ; f_{1}, \cdots, f_{k} ; s\right)$ (for suitable $f_{i}$ ).

(For a reference to the notion of nondegenerate critical point and its index, see e.g. [1].) This might well be regarded as the basic theorem of finite dimensional Morse theory. Morse [23] was concerned with the homology version of this theorem, Bott [1], the homotopy version of 2.1. The proof of 2.1 itself is based on the ideas of the proofs of the weaker statements.

(2.2) Theorem (Morse-Thom). On every closed manifold $W$, there exists a $C^{\infty}$ function with nondegenerate critical points.

For a proof see [41].

By combining 2.1 and 2.2 we see that every manifold can be obtained by attaching handles successively to a disk (we have been restricting ourselves to the compact, empty boundary case only for simplicity).

The main idea of the following theory is to remove superfluous handles (or equivalently critical points) without changing the diffeomorphism type of the manifold. For this one starts (after 2.2) with a "nice function," a function on $M$ given by 2.2 with the additional property that the handles are attached in order according to their dimension (the $s$ in $D^{s} \times D^{n-s}$ ).

(2.3) THEOREM. On every closed manifold there exists a function $f$ with nondegenerate critical points such that at each critical point, the value of $f$ is the index.

This was proved by A. Wallace [44] and the author [38] independently by different methods. (For a general reference to this section see $[34 ; 37]$.)

The actual removing of the extra handles is the main part and for this one needs extra assumptions. The next is the central theorem (or its generalization " 2.4 " to include manifold with boundary).

(2.4) Theorem. Let $M$ be a simply connected closed manifold of dimension $>5$. Then on $M$ there is a nondegenerate (and nice) function with the minimal number of critical points consistent with the homology structure of $M$.

We make more explicit the conclusion of 2.4. Let $\sigma_{i 1}, \cdots, \sigma_{i p(i)}$, $\tau_{i 1}, \cdots, \tau_{i g(i)}, 0 \leqq i \leqq n$, be a set of generators for a direct sum decomposition of $H_{i}(M), \sigma_{i j}$ free, $\tau_{i j}$ of finite order. Then one can ob- 
tain the function of 2.4 with type numbers $M_{i}$ (the number of critical points of index $i)$ satisfying $M_{i}=p(i)+g(i)+g(i-1)$.

The first special case of 2.4 is

(2.5) Theorem. Let $M$ be a simply connected closed manifold of dimension greater than 5 with no torsion in the homology of $M$. Then there exists a nondegenerate function on $M$ with type numbers equal to the betti numbers of $M$.

We should emphasize that 2.4 and 2.5 should be interpreted from the point of view of 2.1. One may apply 2.5 to the case of a "homotopy sphere" (using 2.1 of course).

(2.6) Theorem. Let $M^{n}$ be a simply connected closed manifold with the homology groups of a sphere, $n>5$. Then $M$ can be obtained by "gluing" two copies of the n-disk by a diffeomorphism from the boundary of one to the boundary of the other.

For $n=5$, the theorem is true and can be proved by an additional argument.

Theorem 2.6 implies the weaker statement ("the generalized Poincaré conjecture in higher dimensions") that a homotopy sphere of dimension $\geqq 5$ is homeomorphic to $S^{n}$, see [33]. Subsequently, Stallings [39] and Zeeman [50] found a proof of this last statement.

Theorem 2.4 was developed through the papers $[34 ; 35]$ and appears in the above form in [37]. Rather than try to give an idea of the proof of 2.4, we refer the reader to these papers. One may also refer to $[2 ; 3]$ and $[15]$.

The analogue of 2.4 , say $2.4^{\prime}$ is also proved for manifolds with boundary [37] and this analogue implies the $h$-cobordism theorem stated below. The simplest case of $2.4^{\prime}$ is

(2.7) DisK THEOREM. Let $M^{n}$ be a contractible compact manifold, $n>5, \partial M$ connected and simply connected. Then $M^{n}$ is diffeomorphic to the disk $D^{n}$.

We now discuss another aspect of the preceding theory, the relationship between diffeomorphism and $h$-cobordism. Two oriented, closed manifolds $M_{1}, M_{2}$ are cobordant if there exists a compact oriented manifold $W$ with $\partial W=M_{1}-M_{2}$ (taking into account orientations). Thom in [40] reduced the cobordism classification of manifolds to a problem in homotopy theory which has since been solved.

Two closed oriented manifolds $M_{1}, M_{2}$ are $h$-cobordant (following Milnor [18]) if one can choose $W$ as above so that the inclusions $M_{i} \rightarrow W, i=1,2$ are homotopy equivalences. The idea of $h$-cobordism 
(homotopy-cobordism) is to replace diffeomorphism by a notion of equivalence, a priori weaker than diffeomorphism and amenable to study using homotopy and cobordism theory.

The following theorem was proved in [37], with special cases in $[34 ; 35]$. It is also a consequence of $2.4^{\prime}$.

(2.8) The $h$-COBORdism THEOREM. Let $M_{1}^{n}, M_{2}^{n}$ be closed oriented simply connected manifolds, $n>4$, which are $h$-cobordant. Then $M_{1}$ and $M_{2}$ are diffeomorphic.

Milnor [20] has shown that 2.7 is false for nonsimply connected manifolds. On the other hand Mazur [16] has generalized 2.7 to a theorem which includes the nonsimply connected manifolds.

Theorem 2.8 reduces the diffeomorphism problem for a large class of manifolds to the $h$-cobordism problem. This $h$-cobordism problem has been put into quite good shape for homotopy spheres by Milnor [19], Kervaire and Milnor [12], and recently Novikov [24] has found a general theorem.

Kervaire and Milnor show that homotopy spheres of dimension $n$, with equivalence defined by $h$-cobordism form an abelian group $\mathfrak{H}^{n}$. Their main theorem is the following.

(2.9) ThEOREM. $\mathcal{K}^{n}$ is finite, $n \neq 3$.

Kervaire and Milnor go on to find much information about the structure of this finite abelian group, in particular, to find its order for $4 \leqq n \leqq 18$. The main technique in the proof of 2.9 is what is called spherical modification or surgery, see $[44 ; 22]$.

Putting together 2.8 and 2.9 one obtains a classification of the simplest type of closed manifolds, the homotopy spheres, of dimension $n$ for $5 \leqq n \leqq 18$ and for general $n$ the finiteness theorem. This also gives theorems on differentiable structures on spheres. See [35] or [12].

Most recently, Novikov has found a very general theorem on the $h$-cobordism structure of manifolds (and hence by 2.8 , the diffeomorphism structure). We refer the reader to [24] for a brief account of this.

Lastly we mention some specific results on the manifold classification problem $[36 ; 37]$.

(2.10) Theorem. (a) There is a 1-1 correspondence between simplyconnected closed 5-manifolds with vanishing 2 nd Stiefel-Whitney class and finitely generated abelian groups, the correspondence given by $M \rightarrow$ Free part $\mathrm{H}_{2}(M)+\frac{1}{2}$ Torsion part $\mathrm{H}_{2}(M)$. 
(b) Every 2-connected 6-manifold is diffeomorphic to $S^{6}$ or a sum (in the sense of [18]) of $r$ copies of $S^{3} \times S^{3}, r$ a positive integer.

C. T. C. Wall has very general theorems which extend the above results to $(n-1)$-connected $2 n$-manifolds [43].

3. We give a substantial account of immersion theory because the main problem here has been completely reduced to homotopy theory.

An immersion of one manifold $M^{k}$ in a second $X^{n}$ is a $C^{\infty}$ map $f: M \rightarrow X$ with the property that for each $p \in M$, in some coordinate systems (and hence all) about $p$ and $f(p)$, the Jacobian matrix of $f$ has rank $k$. A regular homotopy is a homotopy $f_{t}: M \rightarrow X, 0 \leqq t \leqq 1$ which for each $t$ is an immersion and which has the additional property that the induced map $F_{t}: T_{M} \rightarrow T_{X}$ (the derivative) on the tangent spaces is continuous (on $T_{M} \times I$ ). One could obtain an equivalent theory by requiring in place of the last property that $f_{t}$ be a differentiable map of $M \times I$ into $X$.

The fundamental problem of immersion theory is: given manifolds $M$ and $X$, find the equivalence classes of immersions of $M$ in $X$, equivalent under regular homotopy. This includes in particular the problem of whether $M$ can be immersed in $X$ at all. This general problem is in good shape. The complete answer has been given recently in terms of homotopy theory as we shall see.

The first theorem of this type was based on "general position" arguments and proved by Whitney [46] in 1936.

(3.1) ThEOREM. Given manifolds $M^{k}, X^{n}$, any two immersions $f, g: M \rightarrow X$ which are homotopic are regularly homotopic if $n \geqq 2 k+2$. If $n \geqq 2 k$ there exists an immersion of $M$ in $X$.

Recent proofs of the second part of this theorem can be found in [17] and [28].

The first statement of 3.1 is equally true with $2 k+2$ replaced by $2 k+1$. Most of the theorems in this survey on the existence of immersions and imbeddings can be strengthened with an approximation property of some sort. Although these are important, for simplicity we omit them.

The first immersion theorem for which arguments transcending general position are needed was the Whitney-Graustein theorem [45] (proved in a paper by Whitney who gives much credit to Graustein). For an immersion $f: S^{1} \rightarrow E^{2}, S^{1}, E^{2}$ oriented, the induced map on the tangent vectors yields a map of $S^{1}$ into $S^{1}$; the degree (an integer) of this map times $2 \pi$ is called by Whitney the rotation number.

(3.2) Theorem (Whitney-Graustein). Two immersions of $S^{1}$ in 
$E^{2}$ are regularly homotopic if and only if they have the same rotation number. There exists an immersion of $S^{1}$ in $E^{2}$ with prescribed rotation number of the form an integer times $2 \pi$.

The next step in the theory of immersions was again taken by Whitney [48] in 1944 with the following theorem.

(3.3) Theorem. Every $k$-manifold can be immersed in $E^{2 k-1}$ for $k>1$.

The proof of this is quite difficult and involved a careful analysis of the critical points of a differentiable mapping of $M^{k}$ into $E^{2 k-1}$. In this dimension, these critical points are isolated in a suitable approximation of a given map, but have to be removed to obtain an immersion. The difficulties in the study of singularities of differentiable maps have limited this method, although, very recently, Haefliger [4] has used effectively Whitney's ideas in the above proof, both in studying imbeddings and immersions. We shall say more about this later.

Some of the recent progress in imbeddings and immersions can be measured by Whitney's statement in the above paper. "It is a highly difficult problem to see if the imbedding and immersion theorems of the preceding paper and the present one can be improved upon." $\mathrm{He}$ goes on to ask if every open or orientable $M^{4}$ may be imbedded in $E^{7}$ and immersed in $E^{6}$. The complex projective plane cannot be immersed in $E^{6}$, but every open $M^{4}$ can be imbedded in $E^{7}$. See Hirsch $[8 ; 9]$. Whitney finally asks if every $M^{3}$ can be imbedded in $E^{5}$. Hirsch has proved this is so if $M^{3}$ is orientable [10].

The next progress in the subject of immersions occurred in papers $[30 ; 31]$ and $[32]$ of the author in 1957-1959. The first generalized the Whitney-Graustein theorem for circles immersed in the plane to circles immersed in an arbitrary manifold, and here methods were introduced which soon led to the solution of the general problem mentioned previously.

Consider " based" immersions of $S^{1}=\{0 \leqq \theta \leqq 2 \pi\}$ in a manifold $X$, that is those which map $\theta=0$ into a fixed point $x_{0}$ of $X$ and the positive unit tangent at $\theta=0$ into a fixed tangent vector of $X$ at $x_{0}$. To each based immersion of $S^{1}$ in $X$, the differential of the immersion associates an element of the fundamental group of $T_{X}^{\prime}$, the unit tangent bundle of $X$.

(3.4) TheOREM. The above is a 1-1 correspondence between (based) regular homotopy classes of based immersions of $S^{1}$ in $X$ and $\pi_{1}\left(T_{X}^{\prime}\right)$.

In the next paper [31] corresponding theorems are proved with $S^{1}$ replaced by $S^{2}$. A noteworthy special case of the theorem proved there 
is that any two immersions of $S^{2}$ in $E^{3}$ are regularly homotopic. It is a good mental exercise to check this for a reflection through a plane of $S^{2}$ in $E^{3}$ and the standard $S^{2}$ in $E^{3}$. This check has been carried through independently by A. Shapiro and N. H. Kuiper, unpublished.

In [32], the classification, under regular homotopy, of immersions of $S^{k}$ in $E^{n}$ is given (any $k, n$ ). This can be stated as follows.

If $f, g: S^{k} \rightarrow E^{n}$ are based immersions (i.e., at a fixed point $x_{0}$ of $S^{k}$, $f\left(x_{0}\right)=g\left(x_{0}\right)$ is prescribed and the derivatives of $f$ and $g$ at $x_{0}$ are prescribed and equal) one can define an invariant $\Omega(f, g) \in \pi_{k}\left(V_{n, k}\right)$ where $\pi_{k}\left(V_{n, k}\right)$ is the $k$ th homotopy group of the Stiefel manifold of $k$-frames in $E^{n}$.

(3.5) Theorem. Based immersions $f, g: S^{k} \rightarrow E^{n}$ are (based) regularly homotopic if and only if $\Omega(f, g)=0$. Furthermore, given a based immersion $f: S^{k} \rightarrow E^{n}$ and $\Omega_{0} \in \pi_{k}\left(V_{n, k}\right)$, there is a based immersion $g: S^{k} \rightarrow E^{n}$ such that $\Omega(f, g)=\Omega_{0}$.

The content of Theorem 3.2 is that the homotopy group $\pi_{k}\left(V_{n, k}\right)$ classifies immersions of $S^{k}$ in $E^{n}$. Information on the groups can be found in [25]. An application of this theorem is that immersions of $S^{k}$ in $E^{2 k}$ are classified by the integers if $k$ is even, the correspondence given by the intersection number.

$R$. Thom in [42] has given a rough exposition of the proof of the previous theorem, which contributes to the theory of conceptualizing part of the proof.

M. Hirsch in his thesis [8], using the results of [32], has generalized 3.5 to the case of immersions of an arbitrary manifold in an arbitrary manifold. If $M^{k}$ and $X^{n}$ are manifolds $T_{M}, T_{X}$ their tangent bundles, a monomorphism $\phi: T_{M} \rightarrow T_{X}$ is a fiber preserving map which is a vector space monomorphism on each fiber. For each immersion $f: M \rightarrow X$ the derivative is a monomorphism $\phi_{f}: T_{M} \rightarrow T_{X}$.

(3.6) Theorem. If $n>k$, the map $f \rightarrow \phi_{f}$ induces a 1-1 correspondence between regular homotopy classes of immersions of $M$ in $X$ and (monomorphism) homotopy classes of monomorphisms of $T_{M}$ into $T_{X}$.

In this theorem one can replace homotopy classes of monomorphisms of $T_{M}$ into $T_{X}$ by equivariant homotopy classes (equivariant with respect to the action of $G L(k)$ ) of the associated $k$-frame bundles of $T_{M}$ and $T_{X}$ respectively. Still another interpretation is that the regular homotopy classes of immersions of $M$ in $X$ are in a $1-1$ correspondence with homotopy classes of cross-sections of the bundle associated to the bundle of $k$-frames of $M$ whose fiber is the bundle of 
$k$-frames of $X$. Recently Hirsch (unpublished) has established the theorem for the case $n=k$ provided $M$ is not closed.

Theorem 3.6 includes as special cases all the previous theorems mentioned here on immersions and has the following consequences as well.

(3.7) THEOREM. If $n>k$ and $M^{k}$ is immersible in $E^{n+r}$ with a normal $r$-field, then it is immersible in $E^{n}$. Conversely, if $M^{k}$ is immersible in $E^{n}$ then (trivially) it is immersible in $E^{n+r}$ with a normal $r$-field.

(3.8) Theorem. If $M^{k}$ is parallelizable (admits $k$ independent continuous tangent vector fields), it can be immersed in $E^{k+1}$. Every closed 3-manifold can be immersed in $E^{4}$; every closed 5-manifold can be immersed in $E^{8}$.

Theorem 3.6 is the fundamental theorem of immersion theory. It reduces all questions pertaining to the existence or classification of immersions to a homotopy problem. The homotopy problem, though far from being solved, has been studied enough to yield much information on immersions through Theorem 3.6 as can be seen for example in Theorem 3.8. Most further work on the existence and classification of immersions would thus seem to lie outside of differential topology proper and in the corresponding homotopy problems.

We note that Haefliger [4] has very recently given another very different proof of Theorem 3.6 under the additional assumption $n>3(k+1) / 2$. See also [7].

We return now to discuss very briefly some of the methods used to proved the theorems of the previous section. The first step is to introduce function spaces of immersions. If $M^{k}, X^{n}$ are manifolds, let $\operatorname{Im}^{r}(M, X)$ be the space of all immersions of $M$ in $X$ endowed with the $C^{r}$ topology, $1 \leqq r \leqq \infty$. This means roughly that two immersions are close if they are pointwise close and their first $r$ derivatives are close. Of course $\operatorname{Im}^{r}(M, X)$ might be empty! A point in $\operatorname{Im}^{r}(M, X)$ is an immersion of $M$ in $X$ and an arc in $\operatorname{Im}^{1}(M, X)$ is a regular homotopy, so the main problem amounts to finding the arc-components of $\operatorname{Im}^{1}(M, X)$ or $\pi_{0}\left(\operatorname{Im}^{1}(M, X)\right)$. One now generalizes the problem to finding not only $\pi_{0}\left(\operatorname{Im}^{1}(M, X)\right)$, but all the homotopy groups of $\operatorname{Im}^{1}(M, X)$. The homotopy groups of $\operatorname{Im}^{r}(M, X)$ do not depend on $r$ and we sometimes omit it. To find these homotopy groups one uses the exact homotopy sequence of a fiber space and one of the main problems becomes, to show certain maps are fiber maps.

The following in fact is perhaps the most difficult part of [32].

(3.9) Theorem. Define a map $\pi: \operatorname{Im}^{2}\left(D^{k}, E^{n}\right) \rightarrow \operatorname{Im}^{2}\left(\partial D^{k}, E^{n}\right)$ by 
restricting an immersion of $D^{k}$ to the boundary. If $n>k+1, \pi$ has the covering homotopy property.

Actually, one uses an extension of this theorem to the case where boundary conditions involving first order derivatives are incorporated into the range space of $\pi$. Since it was first proved, Theorem 3.9 has been generalized and strengthened. The general version, due to Hirsch and Palais [11] is as follows.

(3.10) Theorem. Let $V$ be a submanifold of a manifold $M, X$ another manifold and $\pi: \operatorname{Im}(M, X) \rightarrow \operatorname{Im}(V, X)$ defined by restriction. Then $\pi$ is a fiber map in the sense of Hurewicz (and hence has the covering homotopy property).

A version of 3.10 is also proved with the boundary conditions mentioned above.

An idea not present in the author's original proof of 3.9, but introduced by Thom in [42], was to prove theorems of type 3.9 and 3.10 by first explicitly proving the corresponding theorem for spaces of imbeddings, this theorem being much easier and quite useful itself. The final version of this intermediate result is due to Palais [26].

(3.11) Theorems. Let $M$ be a compact manifold, $V$ a submanifold, and $X$ any manfold. Let $\varepsilon(M, X), \varepsilon(V, X)$ be the respective spaces of imbeddings with the $C^{r}$ topology, $1 \leqq r \leqq \infty$, and $\pi: \mathcal{E}(M, X) \rightarrow \mathcal{E}(V, X)$ defined by restriction. Then $\pi$ is a locally trivial fiber map.

Using Theorems 3.9, 3.10 and an induction basically derived from the fact that the dimension of the boundary of a manifold is one less than the manifold itself, one obtains weak homotopy equivalence theorems. The most general one is due to Hirsch and Palais [11]. Given manifolds $M, X$ let $K(M, X)$ be the space of monomorphisms of $T_{M}$ into $T_{X}$ with the compact open topology. Then as described in $\S 2$, there is a map

$$
\alpha: \operatorname{Im}(M, X) \rightarrow K(M, X) .
$$

(3.12) TheOREM. The map $\alpha$ induces an isomorphism on all the homotopy groups (is a weak homotopy equivalence) if $\operatorname{dim} X>\operatorname{dim} M$.

Theorem 3.12 applied to the zeroth homotopy groups or arc-components of $\operatorname{Im}(M, X)$ and $K(M, X)$ yields Theorem 3.6. Theorem 3.12 was first proved for $\operatorname{Im}\left(S^{k}, E^{n}\right)$ in [32].

4. An imbedding (or differentiable imbedding) is an immersion which is also a homeomorphism onto its image. A regular (or differentiable) isotopy is a regular homotopy which at each stage is an im- 
bedding. The fundamental problem of imbedding theory is; given manifolds $M^{k}, X^{n}$, classify the imbeddings of $M$ in $X$ under equivalence by regular isotopy. This includes the problem: does there exist an imbedding of $M$ in $X$ ? Our discussion of imbedding theory is limited to work on this problem. The difficulty of the general problem is indicated by the special case of imbeddings of $S^{1}$ in $E^{3}$. This problem of classifying "classical" knots is far from being settled (and of course we omit any discussion of this special case although it could well be considered within the scope of differential topology).

Again the first theorems are due to Whitney in 1936 and are proved by general position arguments [46].

(4.1) Theorem. A manifold $M^{k}$ can always be imbedded in $E^{2 k+1}$. Any two homotopic imbeddings of $M$ in $X^{2 k+3}$ are regularly isotopic.

One can replace $X^{2 k+3}$ by $X^{2 k+2}$ here.

See [17] or [28] for recent proofs of the first statement of 6.1.

In 1944, Whitney proved the much harder theorem [47].

(4.2) Theorem. Every k-manifold can be imbedded in $E^{2 k}$.

The methods used in this paper have been important in subsequent developments in imbedding theory. A. Shapiro, in fact, has considerably developed Whitney's ideas in the framework of obstruction theory. Only the first stage of Shapiro's work is in print [29]. Besides being mostly unpublished, the theory has the further disadvantage from our point of view that it is a theory of imbedding for complexes and does not directly apply to give imbeddings (differentiable) of manifolds. On the other hand, Shapiro's work has in part inspired the important theorems of Haefliger that we will come to shortly.

$\mathrm{Wu}$ Wen Tsun in a number of papers, see e.g. [49], has a theory of imbedding and isotopy of complexes which overlaps with Shapiro's work. Shapiro's (unpublished) theorems on the existence of imbeddings of complexes in Euclidean space seem much stronger than those of $\mathrm{Wu}$ Wen Tsun. On the other hand, Shapiro works with spaces derived from the two-fold product of a space, while $\mathrm{Wu}$ studies stronger invariants derived from the $p$-fold products. Also Wu Wen Tsun not only considers existence of imbeddings but isotopy problems as well, including the following one for the differentiable case [49]. The proof is based on Whitney's paper [47].

(4.3) Theorem. Any two imbeddings of a connected manifold $M^{k}$ in $E^{2 k+1}$ are regularly isotopic. 
Haefliger [4] has taken a big step forward in the theory of imbeddings with the following theorems, proved by strong extensions of the work of Whitney, Shapiro and Wu Wen Tsun. Haefliger's main theorem can be expressed as follows.

An imbedding $f: M \rightarrow E^{n}$ induces a map $\phi_{f}: M \times M-M \rightarrow S^{n-1}$ $\left(M \times M-M\right.$ is the product with the diagonal deleted) by $\phi_{f}(x, y)$ $=(f(x)-f(y)) /\|f(y)-f(x)\|$. Then clearly $\phi_{f}$ is equivariant with respect to the involution on $M \times M-M$ which interchanges factors and the antipodal map of $S^{n-1}$.

(4.4) Theorem. If $n>3(k+1) / 2$, the map $f \rightarrow \phi_{f}$ induces a 1-1 correspondence between regular isotopy classes of $M$ in $E^{n}$ and equivariant homotopy classes of $M \times M-M$ into $S^{n-1}$.

The equivariant homotopy classes are in a 1-1 correspondence with homotopy classes of cross-sections of the following bundle $E$. Let $M^{*}$ be the quotient space of $M \times M-M$ under the above involution. The two involutions described above define an action of the cyclic group of order two on $(M \times M-M) \times S^{n-1}$. The orbit space of this action is our bundle $E$ with base $M^{*}$ and fiber $S^{n-1}$.

Haefliger actually proves 4.4 with $E^{n}$ replaced by an arbitrary manifold $X^{n}$.

Another of Haefliger's theorems is the following.

(4.5) Theorem. If $M^{k}$ and $X^{n}$ are manifolds which are respectively $(r-1)$-connected, $r$-connected and $n \geqq 2 k-r+1$ then

(a) if $2 r<n$, any continuous map of $M$ in $X$ is homotopic to an imbedding;

(b) if $2 r<n+1$, two homotopic imbeddings of $M$ in $X$ are regularly isotopic. Thus if $n>3(k+1) / 2$, any two imbeddings of $S^{k}$ in $E^{n}$ are regularly isotopic.

Hirsch has proved some theorems on the existence of imbeddings of manifolds in Euclidean space. Perhaps the most interesting is the following [10].

(4.6) Theorem. Every orientable 3-manifold can be imbedded in $E^{5}$.

We do not discuss here, in general, the highly unstable problem of imbeddings of $M^{k}$ in $X^{n}$ where $n \leqq k+2$ except to mention that 2.7 is relevant to the differentiable Schonflies problem.

Since this section was first written Haefliger has obtained several further important results on imbeddings; see [5]. 


\section{REFERENCES}

1. R. Bott, The stable homotopy of the classical groups, Ann. of Math. (2) 70 (1959), 313-337.

2. H. Cartan, Seminar, 1961-1962, Paris.

3. J. Cerf, Travaux de Smale sur la structure des varietes, Seminaire Bourbaki, 1961-1962, No. 230, Paris.

4. A. Haefliger, Differentiable imbeddings, Bull. Amer. Math. Soc. 67 (1961), 109-112.

5. - Knotted (4k-1)-spheres in 6k-space, Ann. of Math. (2) 75 (1962), $452-466$.

6. - Plongements differentiables de variete dans varietes, Comment. Math. Helv. 36 (1962).

7. A. Haefliger and M. Hirsch, Immersions in the stable range, Ann. of Math. (2) 75 (1962), 231-241.

8. M. Hirsch, Immersions of manifolds, Trans. Amer. Math. Soc. 93 (1959), 242-276.

9. - On imbedding differentiable manifolds in Euclidean space, Ann. of Math. (2) 73 (1961), 566-571.

10. - The imbedding of bounding manifolds in Euclidean space, Ann. of Math. (2) 74 (1961), 494-497.

11. M. Hirsch and R. Palais, unpublished.

12. M. Kervaire and J. Milnor, Groups of homotopy spheres. I, Ann. of Math. (2) (1963) (to appear).

13. S. Lefschetz, Topology, Amer. Math. Soc. Colloq. Publ. Vol. 12, Amer. Math. Soc., Providence, R. I., 1930.

14. H. Levine, Singularities of differentiable mappings. I, Mathematisches Institut der Universität, Bonn, 1959.

15. B. Mazur, The theory of neighborhoods, Harvard University.

16. - Simple neighborhoods, Bull. Amer. Math. Soc. 68 (1962), 87-92.

17. J. Milnor, Differential topology, Princeton, 1959.

18. - Sommes de variêtés différentiables et structures différentiables des sphères, Bull. Soc. Math. France 87 (1959), 439-444.

19. - Differentiable manifolds which are homotopy spheres, Princeton, 1959.

20. - Two complexes which are homeomorphic but combinatorial distinct, Ann. of Math. (2) 74 (1961), 575-590.

21. - On cobordism, a survey of cobordism theory, Enseignement Math. 111 (1962), 16-23.

22. - A procedure for killing homotopy groups of differentiable manifolds, Proc. Sympos. Pure Math. Vol. 3, Amer. Math. Soc., Providence, R. I., 1961.

23. M. Morse, The calculus of variations in the large, Amer. Math. Soc. Colloq. Publ. Vol. 18, Amer. Math. Soc., Providence, R. I., 1934.

24. S. P. Novikov, Diffeomorphisms of simply connected manifolds, Dokl. Akad. Nauk SSSR 143 (1962), 1046-1049=Soviet Math. Dokl. 3 (1962), 540-543.

25. G. F. Paechter, The groups $\Pi_{r}\left(V_{n, m}\right)$. I, Quart. J. Math. Oxford Ser. (2) 7 (1956), 249-268.

26. R. Palais, Local triviality of the restriction map for embeddings, Comment. Math. Helv. 34 (1960), 305-312. 1953.

27. H. Poincaré, Analysis situs, Collected Works, Vol. 6, Gauthier-Villars, Paris,

28. L. S. Pontrjagin, Smooth manifolds and their applications in homotopy theory, Trudy Mat. Inst. Steklov 45 (1955)= Amer. Math. Soc. Transl. (2) 11 (1955). 
29. A. Shapiro, Obstructions to the imbedding of a complex in a euclidean space. I. The first obstruction, Ann. of Math. (2) 66 (1957), 256-269.

30. S. Smale, Regular curves on Riemannian manifolds, Trans. Amer. Math. Soc. 81 (1958), 492-512.

31. - A classification of immersions of the two-sphere, Trans. Amer. Math. Soc. 90 (1959), 281-290.

32. - The classification of immersions of spheres in euclidean spaces, Ann. of Math. (2) 69 (1959), 327-344.

33. - The generalized Poincare conjecture in higher dimensions, Bull. Amer. Math. Soc. 66 (1960), 373-375.

34. - The generalized Poincare conjecture in dimensions greater than four, Ann. of Math. (2) 74 (1961), 391-406.

35. - Differentiable and combinatorial structures on manifolds, Ann. of Math. (2) 74 (1961), 498-502.

36. - On the structure of 5-manifolds, Ann. of Math. (2) 75 (1962), 38-46.

37. - On the structure of manifolds, Amer. J. Math. (to appear).

38. - On gradient dynamical systems, Ann. of Math. (2) 74 (1961), 199-206.

39. J. Stallings, Polyhedral homotopy-spheres, Bull. Amer. Math. Soc. 66 (1960), $485-488$.

40. R. Thom, Quelques proprietés globales des variettés différentiables, Comment. Math. Helv. 29 (1954), 17-85.

41. - Les singularités des applications différentiables, Ann. Inst. Fourier (Grenoble) 6 (1956), 43-87.

42. - La classification des immersions d'après Smale, Séminaire Bourbaki, December 1957, Paris.

43. C. T. C. Wall, Classification of (n-1)-connected 2n-manifolds, Ann. of Math. (2) 75 (1962), 163-189.

44. A. H. Wallace, Modifications and cobounding manifolds, Canad. J. Math. 12 (1960), 503-528.

45. H. Whitney, On regular closed curves in the plane, Compositio Math. 4 (1937), $276-284$.

46. - Differentiable manifolds, Ann. of Math. (2) 37 (1936), 645-680.

47. - The self-intersections of a smooth n-manifold in $2 n$ space, Ann. of Math. (2) 45 (1949), 220-246.

48. - The singularities of a smooth n-manifold in (2n-1)-space, Ann. of Math. (2) 45 (1949), 247-293.

49. Wu Wen Tsun, On the isotopy of $C^{r}$ manifolds of dimension $n$ in euclidean $(2 n+1)$-space, Science Records (N. S.) 2 (1958), 271-275.

50. E. C. Zeeman, The generalized Poincare conjecture, Bull. Amer. Math. Soc. 67 (1961), 270.

\section{Columbia University}

